\title{
Challenges of using Personal Data to drive Personalised Electronic Programme Guides
}

\author{
Neelima Sailaja \\ The Mixed Reality Lab \\ University of Nottingham, UK \\ first.last@nottingham.ac.uk
}

\author{
Andy Crabtree \\ The Mixed Reality Lab \\ University of Nottingham, UK \\ first.last@nottingham.ac.uk
}

\author{
Phil Stenton \\ BBC R\&D \\ Salford, UK \\ first.last@bbc.co.uk
}

\begin{abstract}
Media researchers are adopting personalisation in diverse ways to deliver increasingly context-sensitive and customised media experiences. This paper explores user attitudes towards a personalised Electronic Programme Guide which tailors media recommendations based on users' personal data. We used scenario based exploration enabled by the use of probes to convey the functionalities of data-driven Personalised EPGs and to facilitate user discussions around its potential use. Users preferred personalised EPGs over current popular EPGs but expressed a significant lack of trust in the personal data collection that drives personalisation. Users appreciated the functionalities afforded by personalisation of media but were apprehensive about the implications of the personal data being collected about them, particularly in the context of their homes. This calls for the need to design future personalised media experiences that help enhance trust in these socio-technical settings.
\end{abstract}

\section{Author Keywords}

EPG;media;interaction;personal data;focus groups;

\section{ACM Classification Keywords}

H.5.m. Information interfaces and presentation (e.g., HCI): Miscellaneous;

\section{INTRODUCTION}

It is predicted that "over the next two decades we will see a gradual shift away from the mass-production of one-sizefits all generic products and services, in favour of highly personalised services tailored to consumers' individual needs." [31] The broadcast industry is also moving into this domain of personalization with media being tailored using viewers' personal data $[11,18,36]$.

This study extends the idea of personalising media to the case of an Electronic Programme Guide. With the EPG being personalised, it would upgrade from a simple list of

\footnotetext{
Permission to make digital or hard copies of all or part of this work for personal or classroom use is granted without fee provided that copies are not made or distributed for profit or commercial advantage and that copies bear this notice and the full citation on the first page. Copyrights for components of this work owned by others than the author(s) must be honored. Abstracting with credit is permitted. To copy otherwise, or republish, to post on servers or to redistribute to lists, requires prior specific permission and/or a fee. Request permissions from Permissions@acm.org.

CHI 2017, May 06 - 11, 2017, Denver, CO, USA

Copyright is held by the owner/author(s). Publication rights licensed to ACM ACM 978-1-4503-4655-9/17/05 ..\$15.00

DOI: http://dx.doi.org/10.1145/3025453.3025986
}

programmes chosen by the broadcaster to an intelligent mix of both live broadcast and video on demand[15], laid out against a timeline, featuring content of interest to the user and reflecting the user's lifestyle and TV viewing habits.

Recent HCI research in EPGs[7,12,20,35] focus on improvements in EPG user interface design. They have shown that users "found it overwhelming and disliked [.... confusing ordering of channels" [25] and that they "have fundamental difficulties navigating in this way" [25]. It is recommended that "the cognitive demands associated with using an EPG" [42] be reduced by "simplifying the decision making process" [42]. Personalisation would help out here "by limiting the number of channels" [42]. Recommendations in EPGs have been considered before $[23,27,28,39]$ but does not extend beyond technical or cognitive recommendations to the contextual implications of such technologies.

Personalisation entails the collection and analysis of data, often personal data[9]. In the context of media, this data could range from devices used and genres watched to pauses made and repeats requested. With the addition of IoT, the situation is amplified considerably. This has led to a 'crisis in trust' [44] in the digital economy and hence for such innovation to be sustainable, data privacy concerns have to be respected.

Usable privacy has been a significant application domain in $\mathrm{HCI}$ in the recent years[1,5,6,14,26]. Research has consistently highlighted the importance of making the user more involved in the data exchange process through better data legibility and control[32]. Usable privacy research ranging from mobile applications $[29,41]$ to websites[16,43] to IoT[17] reflect these concepts. With media and broadcast growing to be increasingly online, ubiquitous and personalised, there is a need to understand and explore the potential of usable privacy in overcoming the barriers created by this shift in modern media experiences.

This study is a start to undertaking that challenge as it explores user attitudes and orientations towards personalised media, an adaptive EPG, which is driven by users' personal data. We designed EPG UIs which represented different scenarios situated in varying contexts to be used as probes to aid in this exploration. Our results suggest that personalised EPGs are preferred over current EPGs but there is a lack of trust associated with the personal data that would be used to tailor recommendations. 
Users are concerned about data collection happening within their homes and are not satisfied with their current levels of understanding and control over data exchange. HCI research could answer this growing call for the design of media experiences that embody improved privacy measures through increased transparency, better mechanisms for data control and enhanced options for user engagement.

\section{METHODOLOGY}

The methodology adopted for this study was scenario based exploration. It is appropriate in this case as it could be used to elaborate a conceptual model of a future system, "a description of the proposed system in terms of a set of integrated ideas and concepts about what it should do, behave and look like, that will be understandable by the users" [37]. Thus, the focus of design is shifted by placing the highest priority on the user through "defining system operations by describing how people will use a system to accomplish work tasks and other activities." [38] Therefore, software systems are considered to be sociotechnical interventions that are embedded in different social contexts and that aid in shaping how people conduct their jobs, leisure, play, etc. within them.

The scenarios were presented through mockups of the EPG UIs in different social contexts. These mockups were used as probes[24] to explore and understand user orientations towards novel broadcast solutions which deliver tailored content using personal data. The use of probes were appropriate for this study as they "act[ed] as design intervention[s] that elicit inspirational material while avoiding the understood social roles of researchers and researched"[19]. Thus, scenario based exploration through the use of probes helped explore the application of the technology in varying social contexts while empowering the user to validate requirements for the technology by moving them closer to the design space[21].

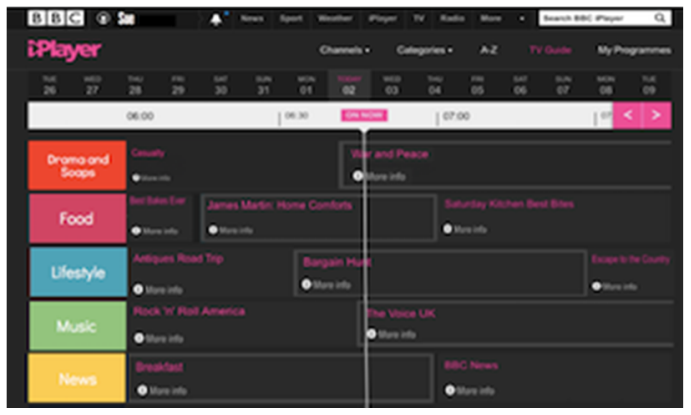

Figure 1 : Recommender version $@$ Neelima Sailaja

\section{Participants}

The study had 20 participants, all above the age of 18 , from varying socio-economic backgrounds and all regular media consumers. Most of them were acquainted with the basic concepts of modern broadcast and media, with 12 of them using an EPG and 17 of them using Video on Demand either daily, weekly or monthly.

\section{Scenarios}

The study used four scenarios, conveyed using UI mockups of the EPG. The mockups for each case were presented to the participants along with a description of its functionalities. This was followed by a brief discussion on the users' thoughts about the proposed technology which lasted around 30 to 45 minutes. Questionnaires regarding the current TV watching habits of the participants and the proposed EPG functionalities were provided before and after the discussions, respectively. Four focus groups were held, two in Manchester and two in Nottingham.

Scenario 1, Recommender : This case delivers an automated fully filled EPG based on the users' TV viewing history. All content that is not watched would be intelligently filtered out, channel wise grouping of content ( in the current EPG ) would be replaced with category wise grouping, where content from different channels would be grouped to fill up the various categories. ( Figure 1 )

Scenario 2, Calendar metaphor : This EPG follows a calendar metaphor and is built by the user by pulling in content of their choice. It would also be grouped by categories ( which the user creates and customizes ). Previous studies have shown that $77 \%$ of users find new programmes through recommendations from friends[30] and hence we introduced social groups, where users can form groups with other remotely located users and share show recommendations between members of the group.

Scenario 3, Collaborative Profiles : This case accounts for the social nature of TV viewing. It is assumed that content users watch with different social groups would be noticeably different. In order to accommodate this situation, the EPG would give collaborated profiles where content watched with a specific group or person will be available in the profile associated with that group.

Scenario 4, Inclusion of Internet of Things : The introduction of Internet of Things would include devices in the home automatically profiling users to produce accurate media recommendations, adapted to who is watching, at what time, in which room of the house, during what season of the year, etc. The case was demonstrated with the help of a sample profile that would be generated as part of the data collection process.

The data was analysed through the application of thematic analysis driven by grounded theory[8][40]. The process involved open coding of the data followed by extraction of the themes that emerged naturally.

\section{RESULTS}

The results of the discussions show that the users appreciated the advantages afforded by personalisation of EPGs and suggested better support for easier interactions and social viewing. But they also expressed genuine fear and lack of trust associated with the exchange of personal data which drives the technology and was apprehensive 
about adopting such personalization into their lives due to this insecurity.

\section{Need for improved media experience}

\section{Appreciation of personalisation}

The results show a definite appreciation of the personalised EPG. Users wanted the recommendations to go beyond being just a machine output, which was the current practice with systems like Amazon that pushed "incredibly intrusive and phenomenally annoying"[P8] recommendations. They requested for efficient and effective recommendations which would go beyond recommending just Top Gear and suggest specialised recommendations like "70s car chase movies"[P16].

But while users appreciated tailored recommendations they also expected not to be "constrained"[P15] by the system or lose the serendipity of "discover[ing]"[P11] new shows. For these reasons, the availability of the search option on the interface was often noticed and acknowledged.

\section{Need for quick and simple interactions}

Users wanted the interactions with the EPG to be fast and simple and so liked the idea of ease of navigation through the elimination of the need to "scroll all the way to the bottom"[P2] since "there is always something to watch [instead of] empty slots"[P15] or content they are not interested in.

Users mentioned that the calendar version "sounds like a lot of work[P5]" and that they could not "imagine..[]..sitting down with a schedule and sort of saying, I can watch that at 8 p.m. and I can watch that at 9 p.m[P18]". So the idea of planning TV viewing using something like a calendar was considered "a lot of effort [...] in setting [...] up with very little gain compared to the .[]. recommender system"[P20]. This further emphasises the need for quick and easy interactions with the experience.

\section{Support for social viewing}

The relation between media consumption and the social life of the users was shown to be rich. Users showed genuine interest in aspects of the technology that enabled easier integration of social viewing both in co-located and remote scenarios.

In situations of co-located viewing, users considered the concept of collaborative profiles especially helpful. They thought it would help moderate tensions that arise while deciding what content to watch. Parents thought it would help while watching TV with children. P17 who lives in a shared house and does not interact much with her housemates except while watching TV in the common room expressed that it "not only [provides] the opportunity of recommending the programme that fits everyones' taste but it also [...] let[s] other people know this person dislikes these kind of programmes"[P17].
While they were not watching TV with someone, users expressed the need to share what they were watching with friends and family and so they liked the idea of forming private social groups where recommendations could be shared between members of the group. The idea of "orchestrating shared viewing"[P7] or as P6 puts it, "it could be like a WhatsApp group like oh... we are watching this tonight and put it in the thing and we can see if they watched it or not" was considered useful in enhancing the experience. Users like having the capability to share in private groups versus through established social media as they consider public shares to "often [be] very irritating for other people."[30]

\section{Mistrust associated with personal data exchange}

\section{Privacy issues and fear}

Users' acknowledgement of the value presented by personalised media was often overshadowed by their fear associated with personal data exchange. This can be noticed in comments like "I have got privacy issues, I'm not ready to give out data just to get better recommendations of programmes I watch"[P20].

The use of IoT instigated predictions of dystopian scenarios like "so its detecting who I am and say I'm watching a lot of new programmes about terrorism what guarantee do I have that this information isn't going to put me on a watch list and suddenly the camera is watching you all the time, its detecting anger when I'm watching programmes about terrorism. I mean this sort of thing could easily be interpreted in ways that would have impact on greater scrutiny" [P16].

\section{Lack of trust in the context of the home}

The fear and reluctance to share data was often traced to users' lack of confidence in the corporations rather than the technology itself. Users thought "[the companies] are going to be selling where I am, what I'm doing"[P7] "to third parties"'[P11].

This is particularly perturbing because media consumption is often associated with the home. And since data is relational[13] and hence data about one person often involves data about other associated individuals, media data in the home was repeatedly associated with children. Users expressed concerns like "my kids, are you recording my kids? [....] I'd be freaking out"[P11]. Generally, the idea of being able to record one's media habits, especially while they are home was received with a lack of trust and discomfort as shown in the following quotes. "That creeps me out"[P12], “Thats super creepy."[P14] and "It gets slightly spooky"[P12].

\section{Need for better legibility and control}

Users attributed this genuine paucity of trust in the present day data economy to the current lack of transparency in data exchange mechanisms. They mostly requested for 
transparency through dashboards and visualisations so that they "actually ...[...]... know what is happening" [P15].

Users claimed that providing more transparency and control helps improve trust in the organisation and foster a better data exchange scenario, as currently the only option is to "spend hours setting up the privacy controls in Facebook and in the end, even if you have the best privacy settings on Facebook, you still have to somehow trust Facebook to actually do it"[P18].

\section{DISCUSSION}

From the results of the study, the challenges facing the use of personal data in driving personalised EPGs could be classified into four.

Firstly, it is the need for future media to be able to accommodate and support users' offline social interactions[30]. While our proposal of collaborative profiles and formation of social groups are two potential solutions, the HCI community could help broaden this research by looking further into alternatives that allow better integration of the television experience into users' social lives. This could be through measures like EPG collaboration with the users' calendar, non-intrusive social media involvement, novel methods of sharing content through mobile devices etc.

Secondly, it is the lack of trust users show in the collection and use of personal data, particularly in the context of the home. The home does not often consist of a single, uniform, homogenous cohort of users, it is a mix of user groups which might include parents, children, guests, lodgers etc. The use of personal data comes with a different set of implications for each of these cohorts which has resulted in the need for data control mechanisms that cater to the needs of the entire spectrum of users. Possible solutions include smartly designed control panels, settings pages or granular control interfaces etc. The EPG could also have alterable, predefined modes for when children or guests are present, which could be initiated with a single click. Timely notifications and nudges[43] that remind users of the amount of data exchanged and that extends to mobile[3] and watch devices for ubiquitous access and control of data is another possible solution towards building ongoing trust. The HCI community could take this up as a challenge by exploring the most effective alternatives that support easy data control mechanisms for diverse cohorts of users.

Thirdly, it is the lack of data transparency afforded by organisations that collect and use personal data. There is a call for better mechanisms that could introduce data transparency and moreover legibility[32] into the system. This could be through the use of appropriate data visualisations, dashboards that display monthly statements of data use and inferences made, or alerts that inform the user of unexpected data usage patterns. HCI researchers could play a key role here by exploring and applying the most efficient solutions that enable better legibility of data collection and use.
Fourthly, the lack of accountability that has resulted from low levels of data transparency and inefficient data control mechanisms. Any party that would consume personal data is accountable to both regulatory bodies and the user. Legislation often expects systems design to provide computational tools to enable accountability. The Consumer Privacy Bill of Rights[22] in the US and the GDPR[45] in the EU, all highlight the need for building accountability into systems that collect and use personal data. But there are many barriers towards getting the users engaged with such privacy enhancing technology, e.g., the privacy paradox[34], hyperbolic discounting[2] etc. The HCI community could help overcome these challenges by making privacy enabling technologies ( like Personal Data Vaults[33] and DataBox[4], that help users manage personal data sharing through applications like the EPG ) more engaging and less intimidating. Another alternative which calls for more attention is incorporating privacy-bydesign[10] into the design and development cycle of systems so that the resulting technology encourages the user to make privacy enhancing choices. $\mathrm{HCI}$, with its multidisciplinary research nature is uniquely positioned to help combine the legal, technical and usability aspects of personal data to ensure successful and sustainable adoption of data driven experiences into users' everyday lives.

\section{CONCLUSION}

This study reports the scenario based exploration of a personalised Electronic Programme Guide. It uncovers the challenges associated with the use of personalised media which is powered by the collection and use of personal data.

The research showed that personalisation of EPGs is preferred over current EPGs due to the higher chances of finding content of interest faster through tailored recommendations. But while users acknowledged the technological advances offered by personalisation, they showed an obvious deficit of trust in sharing their personal data for this purpose. Users felt disconcerted at the possibility of data being collected in their homes and their children's data being recorded. They expressed a genuine lack of trust in organisations when they were not provided with enough information and control over their personal data. In order to successfully deliver users with enhanced media experiences through personalisation, it is necessary to encourage a sustainably trustworthy mechanism for personal data exchange. HCI researchers should recognise this as a priority and help assuage the current user attitudes by designing systems that reflect values of transparency, trust and privacy. 


\section{REFERENCES}

1. MS Ackerman and L Cranor. 1999. Privacy critics: UI components to safeguard users' privacy. CHI'99 Extended Abstracts on Human Factors in Computing Systems: 258-259.

2. Alessandro Acquisti and Jens Grossklags. 2004. Privacy attitudes and privacy behavior. Economics of Information Security: 165-178.

3. H. Almuhimedi. 2015. Your location has been shared 5398 times - A field study on mobile app privacy nudging. In Proceedings of the 33rd Annual ACM Conference on Human Factors in Computing Systems: 787-796.

4. Yousef Amar, Hamed Haddadi, and Richard Mortier. Privacy-Aware Infrastructure for Managing Personal Data Personal Data Arbitering within the Databox Framework. Proceedings of the 2016 conference on ACM SIGCOMM 2016 Conference: $571-572$.

5. I Bilogrevic and M Ortlieb. 2016. If You Put All The Pieces Together...: Attitudes Towards Data Combination and Sharing Across Services and Companies. Proceedings of the 2016 CHI Conference on Human Factors in Computing Systems: 5215-5227.

6. C Birge. 2009. Enhancing research into usable privacy and security. Proceedings of the 27th ACM international conference on Design of communication: 221-226.

7. Sabina Bonnici. 2003. Which channel is that on? A design model for electronic programme guides. Proceedings of the 1st European conference on interactive television: from viewers to actors: 4957.

8. V Braun and V Clarke. 2006. Using thematic analysis in psychology. Qualitative research in psychology 3, 2: 77-101.

9. Pam Briggs. 2013. DR 4: Will an increasing element of our identity be "devolved" to machines? Future Identities: Changing identities in the UK the next 10 years.

10. Ann Cavoukian. 2009. Privacy by Design - The 7 foundational principles - Implementation and mapping of fair information practices. Information and Privacy Commissioner of Ontario, Canada.

11. Tony Churmside. 2013. Object-Based Broadcasting. Retrieved September 10, 2016 from http://www.bbc.co.uk/rd/blog/2013-05-objectbased-approach-to-broadcasting

12. Cyril Concolato. 2009. Generation, Streaming and Presentation of Electronic Program Guide. Proc. European Interactive TV conference, Leuven, Belgium.: 1-4.
13. Andy Crabtree and Richard Mortier. Human Data Interaction : Historical Lessons from Social Studies and CSCW. ECSCW 2015: Proceedings of the 14th European Conference on Computer Supported Cooperative Work, 19-23 September 2015, Oslo, Norway: 3-21.

14. LF Cranor, P Guduru, and M Arjula. 2006. User interfaces for privacy agents. ACM Transactions on Computer-Human Interaction (TOCHI) 13, 2: 135178.

15. DCMS. 2013. Amendments to the Communications Act 2003 (Electronic Programme Guides).

16. S Egelman, M Harbach, and E Peer. 2016. Behavior Ever Follows Intention?: A Validation of the Security Behavior Intentions Scale (SeBIS). In Proceedings of the 2016 CHI Conference on Human Factors in Computing Systems: 5257-5261.

17. S Egelman, R Kannavara, and R Chow. 2015. Is this thing on?: Crowdsourcing privacy indicators for ubiquitous sensing platforms. Proceedings of the 33rd Annual ACM Conference on Human Factors in Computing Systems: 1669-1678.

18. Ian Forrester. 2016. Visual Perceptive Media gets people talking. Retrieved September 10, 2016 from http://www.bbc.co.uk/rd/blog/2015-12-visualperceptive-media-gets-people-talking

19. William Gaver. 2001. Cultural Probes- Probing People for Design Inspiration. SIGCHI. DK.

20. Chris Harrison, Brian Amento, and Larry Stead. 2008. iEPG: an ego-centric electronic program guide and recommendation interface. Proceedings of the 1st international conference on Designing interactive user experiences for TV and video. ACM, 2008.: 23-26.

21. Terry Hemmings, Karen Clarke, Mark Rouncefield, Andy Crabtree, and Tom Rodden. 2002. Probing the Probes. PDC, June: 42-50.

22. White House. 2012. Consumer data privacy in a networked world: A framework for protecting privacy and promoting innovation in the global digital economy. White House, Washington, DC: $1-62$.

23. SH Hsu, MH Wen, HC Lin, CC Lee, and CH Lee. 2007. AIMED-A personalized TV recommendation system. European Conference on Interactive Television: 166-174.

24. Hilary Hutchinson, Wendy E. Mackay, Bo Westerlund, et al. 2003. Technology probes: inspiring design for and with families. Proceedings of the SIGCHI Conference on Human Factors in Computing Systems - CHI '03: 17-24. 
25. Owen Daly Jones. 2000. Navigating your TV: The Usability of Electronic Programme Guides. UsableiTV 3 (2002): 2-6 3: 2-6.

26. CM Karat, C Brodie, and J Karat. 2006. Usable privacy and security for personal information management. Communications of the ACM 49, 1: $56-57$.

27. C Krauss, L George, and S Arbanowski. 2013. TV predictor: personalized program recommendations to be displayed on SmartTVs. Proceedings of the 2nd international workshop on big data, streams and heterogeneous source mining: Algorithms, systems, programming models and applications. ACM, 2013.: 63-70.

28. Haesung Lee and Joonhee Kwon. 2013. A Smart TV Application for Personalized Recommender Services. 29, Ca: 174-176.

29. B Liu, MS Andersen, F Schaub, and H Almuhimedi. 2016. Follow My Recommendations: A Personalized Assistant for Mobile App Permissions. Twelfth Symposium on Usable Privacy and Security (SOUPS 2016).

30. J Lull. 1980. The social uses of television. Human communication research 6, 3: 197-209.

31. Mark Curtis. 2016. The "Era of Living Services" and the future of everyday life. Retrieved July 10, 2016 from

http:/www.telegraph.co.uk/sponsored/business/digi tal-leaders/future-series/12208344/the-era-ofliving-services.html

32. Richard Mortier, Hamed Haddadi, Tristan Henderson, Derek Mcauley, and J O N Crowcroft. 2013. Human-Data Interaction: The Human Face of the Data-Driven Society.

33. Min Mun, Shuai Hao, Nilesh Mishra, et al. 2010. Personal data vaults: a locus of control for personal data streams. Proceedings of the 6th International COnference on - Co-NEXT' '10: 17.

34. PA Norberg and DR Horne. 2007. The privacy paradox: Personal information disclosure intentions versus behaviors. Journal of Consumer Affairs 41.1 (2007): 100-126.
35. Marianna Obrist, Christiane Moser, Damien Alliez, Teresa Holocher, and Manfred Tscheligi. 2009. Connecting TV \& PC: an in-situ field evaluation of an unified electronic program guide concept. Proceedings of the seventh european conference on European interactive television conference. ACM, 2009.: 91-100. http://doi.org/10.1145/1542084.1542101

36. M Pike, R Ramchurn, and S Benford. 2016. \# Scanners: exploring the control of adaptive films using brain-computer interaction. Proceedings of the $2016 \mathrm{CHI}$ Conference on Human Factors in Computing Systems: 5385-5396.

37. J Preece, Y Rogers, and H Sharp. 2002. Interaction design.

38. MB Rosson and JM Carroll. 2002. Usability engineering: scenario-based development of human-computer interaction. Morgan Kaufmann.

39. B Smyth and P Cotter. 2000. A personalised TV listings service for the digital TV age. KnowledgeBased Systems 13, 2: 53-59.

40. A Strauss and J Corbin. 1990. Basics of qualitative research. Sage, Newbury Park, CA.

41. J Tan, K Nguyen, and M Theodorides. 2014. The effect of developer-specified explanations for permission requests on smartphone user behavior. Proceedings of the SIGCHI Conference on Human Factors in Computing Systems: 91-100.

42. A Taylor and R Harper. 2002. Switching on to Switch Off: an analysis of routine TV watching habits and their implications for Electronic Programme Guide Design. UsableiTV.

43. Y Wang, PG Leon, A Acquisti, and LF Cranor. 2014. A field trial of privacy nudges for facebook. Proceedings of the SIGCHI conference on human factors in computing systems. ACM: 2367-2376.

44. World Economic Forum and Boston Consulting Group. 2012. Rethinking Personal Data : Strengthening Trust. May: 1-35.

45. Home Page of EU GDPR. Retrieved from http://www.eugdpr.org/ 